\title{
Extension of the target cascading formulation to the design of product families ${ }^{\star}$
}

\author{
M. Kokkolaras, R. Fellini, H.M. Kim, N.F. Michelena, and P.Y. Papalambros
}

\begin{abstract}
The target cascading methodology for optimal product development is extended to product families with predefined platforms. The single-product formulation is modified to accommodate the presence of shared systems, subsystems, and/or components and locally introduced targets. Hierarchical optimization problems associated with each product variant are combined to formulate the product family multicriteria design problem, and common subproblems are identified based on the shared elements (i.e. the platform). The solution of the overall design problem is coordinated so that the shared elements are consistent with the performance and behaviour of the product variants. A simple automotive design example is used to demonstrate the proposed methodology.
\end{abstract}

Key words product platforms, product families, commonality, target cascading, systems design optimization

\section{1}

\section{Introduction}

Product platforms enable rapid enrichment of a product portfolio to meet changing market needs while keeping design and manufacturing cycle times and costs low (Meyer and Lehnerd 1997; Ericsson and Erixon 1999). According to Siddique et al. (1998), McGrath's description of a product platform for high-tech products (McGrath 1999) can be summarized as a collection of the common elements, especially the underlying core technology, implemented across a range of products. Meyer and

Received September 20, 2001

M. Kokkolaras, R. Fellini, H.M. Kim, N.F. Michelena, and P.Y. Papalambros

Department of Mechanical Engineering, The University of Michigan, 2250 G.G. Brown, Ann Arbor, MI 48109-2125, USA e-mail: \{michailk.rfellini, kimhm, nestorm, pyp\}@umich.edu

* This research was presented in part at the keynote address by P.Y. Papalambros at the 3rd ASMO Conference on Engineerig Design Optimization
Lehnerd (1997) elaborated on the term underlying core technology by defining the product platform as the set of parts, interfaces, and manufacturing processes that are shared among a set of products and allow the development of derivative products with cost and time savings. In this manner, it is emphasized that a platform does not only refer to common physical components but also to a common manufacturing and assembly procedure.

A family of products is developed based upon a product platform. Meyer and Lehnerd (1997) define a product family to be a set of individual products that share common technology and address a related set of market applications. It can be argued that the relation between the product platform and the product family is governed by the product architecture. Product architecture is denned by Ulrich and Eppinger (1995) as (a) the arrangement of functional elements, (b) the mapping from functional elements to physical components, and (c) the specification of the interactions among interacting physical components. The same authors classify two main categories of product architectures according to the mapping of functions to components: integral and modular.

Meyer and Lehnerd (1997) discuss strategies for developing robust product platforms by defining the market segmentation grids and then leveraging product design over these segments. They also provide metrics for measuring the platform's efficiency, development cycletime efficiency, commercial effectiveness, and cost price ratio. Ericsson and Erixon (1999) focus on modular product platforms, propose a modular function deployment (MFD) method based on the quality function deployment (QFD) technique, and demonstrate the use of the latter in, among others, automotive industry applications. The MFD method provides a means for identifying the optimal number of parts that the modules should consist of. Moreover, metrics and rules are proposed for evaluating the effects of modularity in terms of development lead time, costs, and capacity, product and systems costs, quality, variant flexibility, upgrading, and recyclability.

Commonality and differentiation indices have been also proposed by Martin and Ishii (1997) within the frame- work of their design for variety methodology. In their approach, the authors take into account manu- 
facturability and propose late-point differentiation concepts for efficient product platform design. In their latest work, Martin and Ishii (2000) differentiate between variety within the current product line and variety across generations of products. Kota and Sethuraman (1998) present a product line commonality index and present a method for benchmarking product families.

Simpson et al. (1999) propose a method for the synthesis and exploration of product platform concepts based on the market segmentation grid and lever aging/ scaling concepts of Meyer and Lehnerd (1997) and the solution of compromise decision support problems using goal programming. Conner et al. (1999) discuss the fact that many metrics proposed by other researchers are accounting measures for efficiency rather than engineering measures for performance. They propose a method for evaluating platform design alternatives and tradeoffs between commonality and individual product performance by means of non-commonality and performance deviation indices and goal programming. However, they recognize that their method makes possibly misleading assumptions in regard to manufacturing and assembly criteria.

Gonzalez-Zugasti et al. (1998) formulate product platform design as a general optimization problem taking into account both performance requirements and cost, and implement the latter as an interactive negotiation model. They recognize some flaws in their method, namely, the subjective choice of weights when defining the objective function, the lack of tradeoffs evaluation, and the possibly vast size of the combinatorial problem. In an extension of that work, Gonzalez-Zugasti and Otto (2000) present a method for determining design specifications of modules and their best combination, assuming a fixed architecture. They account for family, individual, sharing, and compatibility constraints, and use genetic algorithms for solving the general optimization problem. In a similar approach, Pujita et al. (1998) propose a framework for assessing optimality and sensitivity of product platforms including cost models for development and production. They consider a quite simple application and emphasize the need for further developing the mathematical approach.

Siddique et al. (1998) examine the applicability of product variety concepts to automotive design. In particular, they investigate whether product variety design concepts such as standardization, delayed differentiation, modularity, module interfaces, robustness, and mutability can be utilized. Keeping in mind that they limit their consideration for platform only to the underbody structure of a vehicle, they come to the conclusion that some of these concepts cannot be applied, mainly because of the integral nature of its architecture. However, they do mention the possibility of partitioning the underbody platform into major manufacturable and assem-blable modules.

The research work presented so far refers to efforts for developing methods and tools for designing and evaluating product platforms and families. Some are philosophi- cal frameworks that address design issues in a heuristical manner, and some are more mathematical in nature. The development of a product family depends on an appropriate design strategy that addresses commonality, i.e. the platform. Moreover, different product variants are typically characterized by conflicting performance criteria. A multicriteria optimization formulation can identify the associated tradeoffs by means of Pareto surfaces, with commonality constraints used to define the common elements of the various product variants (Nelson et al. 1999; Fellini et al. 2000). Design decisions can then be made based on subjective engineering, marketing, and manufacturing criteria.

Although contributing significantly to the development of design methodologies for product platforms and families, none of the aforementioned methods can both evaluate tradeoffs between family and individual design targets and determine design specifications for the components, given a predefined platform architecture. The target cascading methodology, extended and applied to product family design, aims at filling this gap.

The article is organized as follows. The target cascading methodology for single optimal product development is introduced in the next section, extended for product family development in Sect. 3, and implemented for a family of vehicles in Sect. 4. Results are presented and discussed in Sect. 5, and conclusions are drawn in Sect. 6.

\section{2}

\section{Target cascading for optimal product development}

The development of any complex product is strongly associated with setting and enforcing proper specifications for each of the product's attributes. Analytical target cascading (Kim 2001) is a methodology for the design of large engineering systems at the early product development stages. First, the design problem is partitioned into a hierarchical set of subproblems associated with systems, subsystems, and components. Design specifications (or targets) are defined at the top level of the multilevel design formulation and "cascaded down" to lower levels. Design subproblems are formulated at each level so that components, subsystems, and systems are designed to match the cascaded targets consistent with the overall system targets. The main benefits of target cascading are reduction in design-cycle time, avoidance of design iterations late in the development process, and increased likelihood that physical prototypes will be closer to production quality. Target cascading also facilitates concurrency in system design: Once targets are identified for systems, subsystems, and components, these elements can be isolated and designed in detail independently, allowing the outsourcing of subsystems and components to suppliers. Target cascading offers a robust framework for multilevel design and has been demonstrated to be convergent, 
whereas other similar problem formulations exhibit convergence difficulties.

The analytical target cascading process was presented by Kim et al. (2000) in the context of automotive engineering systems. In this article a more general notation is introduced, from which the design problem for each element (i.e. system, subsystem, or component) can be recovered as a special case. Moreover, the formulation presented herein allows for design specifications to be introduced not only at the top level for the overall product, but also "locally" to account for individual system, subsystem, and/or component requirements. To represent the hierarchy of the partitioned design problem, the set $\mathcal{E}_{i}$ is defined at each level $i$, in which all the elements of the level are included. For each element $j$ in the set $\mathcal{E}_{i}$, the set of children $C_{i j}$ is defined, which includes the elements of the set $\mathcal{E}_{i+1}$ that are children of the element. An illustrative example is presented in Fig. 1. At level $i=1$ of the partitioned problem we have $\mathcal{E}_{2}=\{\mathrm{A}, \mathrm{B}\}$, and for element "B" on that level we have $\mathcal{C}_{2 \mathrm{~B}}=\{\mathrm{C}, \mathrm{D}\}$.

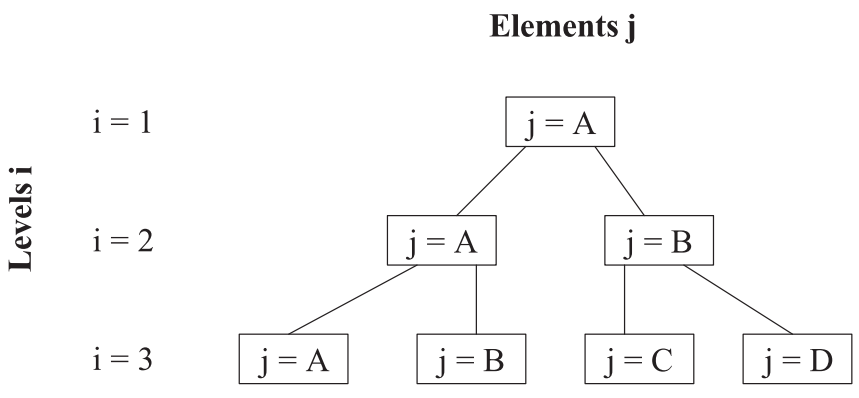

Fig. 1 Example of single product hierarchically partitioned design problem

There are two types of responses: responses $\tilde{R}$ linked to "local" targets (e.g. at the top level), and responses $R$ linked to "cascaded" targets, i.e. linking two successive levels in the problem hierarchy. The design problem $P_{i j}$ corresponding to the $j$-th element at the $i$-th level is formulated as follows:

$\min _{\bar{x}_{i j}}\left\|\tilde{R}_{i j}-T_{i j}\right\|+\left\|R_{i j}-R_{i j}^{U}\right\|+\left\|y_{i j}-y_{i j}^{U}\right\|+$

$\epsilon_{i j}^{R}+\epsilon_{i j}^{y}$

subject to

$$
\begin{aligned}
& \sum_{k \in \mathcal{C}_{i j}}\left\|R_{(i+1) k}-R_{(i+1) k}^{L}\right\| \leq \epsilon_{i j}^{R} \\
& \sum_{k \in \mathcal{C}_{i j}}\left\|y_{(i+1) k}-y_{(i+1) k}^{L}\right\| \leq \epsilon_{i j}^{y} \\
& g_{i j}\left(\hat{R}_{i j}, x_{i j}, y_{i j}\right) \leq 0, \quad h_{i j}\left(\hat{R}_{i j}, x_{i j}, y_{i j}\right)=0,
\end{aligned}
$$

where $\quad \hat{R}_{i j}=\left[\tilde{R}_{i j}, R_{i j}\right]^{T}=r_{i j}\left(R_{(i+1) k_{1}}, \ldots, R_{(i+1) k_{c_{i j}}}\right.$, $\left.x_{i j}, y_{i j}\right), C_{i j}=\left\{k_{1}, \ldots, k_{c_{i j}}\right\}$, and $c_{i j}$ is the number of child elements. Note that an element's response depends on the element's design variables as well as on its children's responses. In the above problem formulation,

$-\bar{x}_{i j}=\left[x_{i j}, y_{i j}, y_{(i+1) k_{1}}, \ldots, y_{(i+1) k_{c_{i j}}}, R_{(i+1) k_{1}}, \ldots\right.$, $\left.R_{(i+1) k_{c_{i j}}}, \epsilon_{i j}^{R}, \epsilon_{i j}^{y}\right]^{T} \in \mathbb{R}^{z_{i j}}$ is the vector of all optimization variables where $z_{i j}:=n_{i j}+l_{i j}+2+$ $\sum_{k \in \mathcal{C}_{i j}} \ell_{(i+1) k}+\sum_{k \in \mathcal{C}_{i j}} d_{(i+1) k}$,

- $x_{i j} \in \mathbb{R}^{n_{i j}}$ is the vector of local design variables, that is, variables exclusively associated with the element,

$-y_{i j} \in \mathbb{R}^{\ell_{i j}}$ is the vector of linking design variables, that is, variables associated with two or more elements that share the same parent,

- $\hat{R}_{i j} \in \mathbb{R}^{\hat{d}_{i j}}$ is the vector of responses, where $\tilde{R}_{i j}$ corresponds to responses linked to local targets, while $R_{i j}$ corresponds to responses linked with cascaded targets $\left(\hat{d}_{i j}:=\tilde{d}_{i j}+d_{i j}\right)$. As shown above, $\hat{R}_{i j}$ is a function of local and linking design variables and responses of child elements: $r_{i j}: \mathbb{R}^{a_{i j}} \rightarrow \mathbb{R}^{\hat{d}_{i j}}$, where $a_{i j}=n_{i j}+$ $\ell_{i j}+\sum_{k \in \mathcal{C}_{i j}} d_{(i+1) k}$

- $\epsilon_{i j}^{R} \in \mathbb{R}_{+} \bigcup\{0\}$ is the tolerance optimization variable for coordinating the responses of the children of the element,

- $\epsilon_{i j}^{y} \in \mathbb{R}_{+} \bigcup\{0\}$ is the tolerance optimization variable for coordinating the linking design variables of the children of the element,

- $T_{i j} \in \mathbb{R}^{\tilde{d}_{i j}}$ is the vector of local target values,

- $R_{i j}^{U} \in \mathbb{R}^{d_{i j}}$ is the vector of response values cascaded down to the element from its parent,

- $y_{i j}^{U} \in \mathbb{R}^{\ell_{i j}}$ is the vector of linking design variable values cascaded down to the element from its parent,

- $R_{(i+1) k}^{L} \in \mathbb{R}^{d_{(i+1) k}}$ is the vector of response values cascaded up to the element from its $k$-th child,

- $y_{(i+1) k}^{L} \in \mathbb{R}^{\ell_{(i+1) k}}$ is the vector of linking design variable values cascaded up to the element from its $k$-th child,

- $g_{i j}: \mathbb{R}^{b_{i j}} \rightarrow \mathbb{R}^{v_{i j}}$ and $h_{i j}: \mathbb{R}^{b_{i j}} \rightarrow \mathbb{R}^{w_{i j}}$ are vector functions representing inequality and equality design constraints, respectively, where $b_{i j}=\hat{d}_{i j}+n_{i j}+\ell_{i j}$, and

- $\|\cdot\|$ is some norm; typically, some weighted norm is used for the metrics involving local targets $T_{i j}$ in order to enable trade-off evaluation studies, while the $\ell_{2}$ norm is used in all other cases.

In the above definitions it is assumed for simplicity that all problems are continuous, but the formulation holds if some optimization variables are discrete. In the latter case, suitable optimization algorithms are necessary for the solution of the associated mixed-integer programming problems.

Non-ascent properties for the analytical target cascading process based on hierarchical overlapping coordination convergence theory (Michelena et al. 1999) are 
presented by Kim (2001) under the assumption that (1) is convex. Convergence properties of analytical target cascading will be presented by Park et al. (2001).

3

\section{Target cascading for product family development}

When designing a family of platform-based products, one must identify the tradeoffs that are a consequence of shared systems, subsystems, and/or components. These tradeoffs exist because the products are no longer optimized for their individual performance, but for the family as a whole. The shared elements of the product family influence the performance of the individual products.

A Pareto-based approach that quantifies the aforementioned tradeoffs was proposed by Nelson et al. (1999). The first step in this method is to formulate individual design problems for each product. The problems are then combined into one family problem using a multicriteria optimization formulation that includes the individual product requirements. By introducing an equality commonality constraint one can specify the product elements to be shared. Solving the multicriteria problem for the Pareto set one can then visualize the design tradeoffs, and choose a suitable design.

In order to exploit the product family structure, the design problem is formulated as a hierarchical optimization problem, as investigated by Fellini et al. (2000). The top level problem addresses family attributes, while lower levels address attributes associated with particular elements. To achieve this within the modified target cascading formulation presented in this article, family targets are defined at the top level and locally introduced targets are defined at lower levels (e.g. the product level) to satisfy individual requirements. Given a set of family and individual product targets, analysis models for all design elements, and a predefined platform, targets are cascaded to elements lower in the hierarchy (i.e. system, subsystem, and component targets are determined).

The rest of this section discusses modifications to the single-product target cascading formulation necessary for the design of product families. These modifications enable subproblems to return design response and linking variable values to multiple parents. Figure 2 illustrates a simple example of a product family with two product variants; each variant is partitioned into two systems, and the two variants share one system.

Desired product family design specifications are defined as targets at the top level and cascaded to lower levels. Product variant targets are introduced at the product level. Design subproblems are formulated at each level so that components, subsystems, and systems are designed to match the cascaded targets while the overall system is consistent. To allow for elements to be shared (i.e. to have multiple parents), the set of parents $\mathcal{P}_{i j}$ is defined for each element $j$ of the set $\mathcal{E}_{i}$ at every level $i$ this set includes the

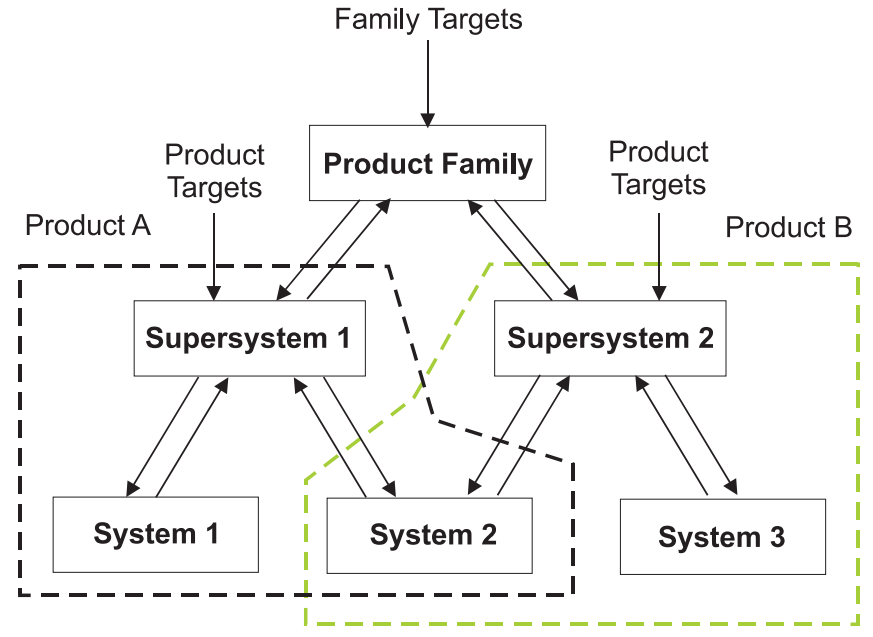

Fig. 2 Example of product family hierarchically partitioned optimal design problem

elements of the set $\mathcal{E}_{i-1}$ that are parents of this element. The design problem $P_{i j}$ for the $j$-th element at the $i$-th level is reformulated as follows:

$$
\begin{aligned}
& \min _{\bar{x}_{i j}}\left\|\tilde{R}_{i j}-T_{i j}\right\|+\sum_{q \in \mathcal{P}_{i j}}\left\|R_{i j}-R_{i j q}^{U}\right\|+ \\
& \sum_{q \in \mathcal{P}_{i j}}\left\|y_{i j}-y_{i j q}^{U}\right\|+\epsilon_{i j}^{R}+\epsilon_{i j}^{y}
\end{aligned}
$$

subject to

$$
\sum_{k \in \mathcal{C}_{i j}}\left\|R_{(i+1) k}-R_{(i+1) k}^{L}\right\| \leq \epsilon_{i j}^{R}
$$

$\sum_{k \in \mathcal{C}_{i j}}\left\|y_{(i+1) k}-y_{(i+1) k}^{L}\right\| \leq \epsilon_{i j}^{y}$

$g_{i j}\left(\hat{R}_{i j}, x_{i j}, y_{i j}\right) \leq 0, \quad h_{i j}\left(\hat{R}_{i j}, x_{i j}, y_{i j}\right)=0$,

with $\mathcal{P}_{i j}=\left\{q_{1}, \ldots, q_{p_{i j}}\right\}$, where $p_{i j}$ is the number of parent elements, and where

- $R_{i j q}^{U} \in \mathbb{R}^{d_{i j}}$ is the vector of response values cascaded to the element from its $q$-th parent,

- $y_{i j q}^{U} \in \mathbb{R}^{l_{i j}}$ is the vector of linking design variable values cascaded to the element from its $q$-th parent.

It can be readily shown that the target cascading formulation for optimal single product design shown in (1) is recovered if all the sets of parents $\mathcal{P}_{i j}$ consist of only one element.

\section{4}

\section{Case study}

To illustrate the use of target cascading for designing a family of products, a multi-vehicle design problem has 


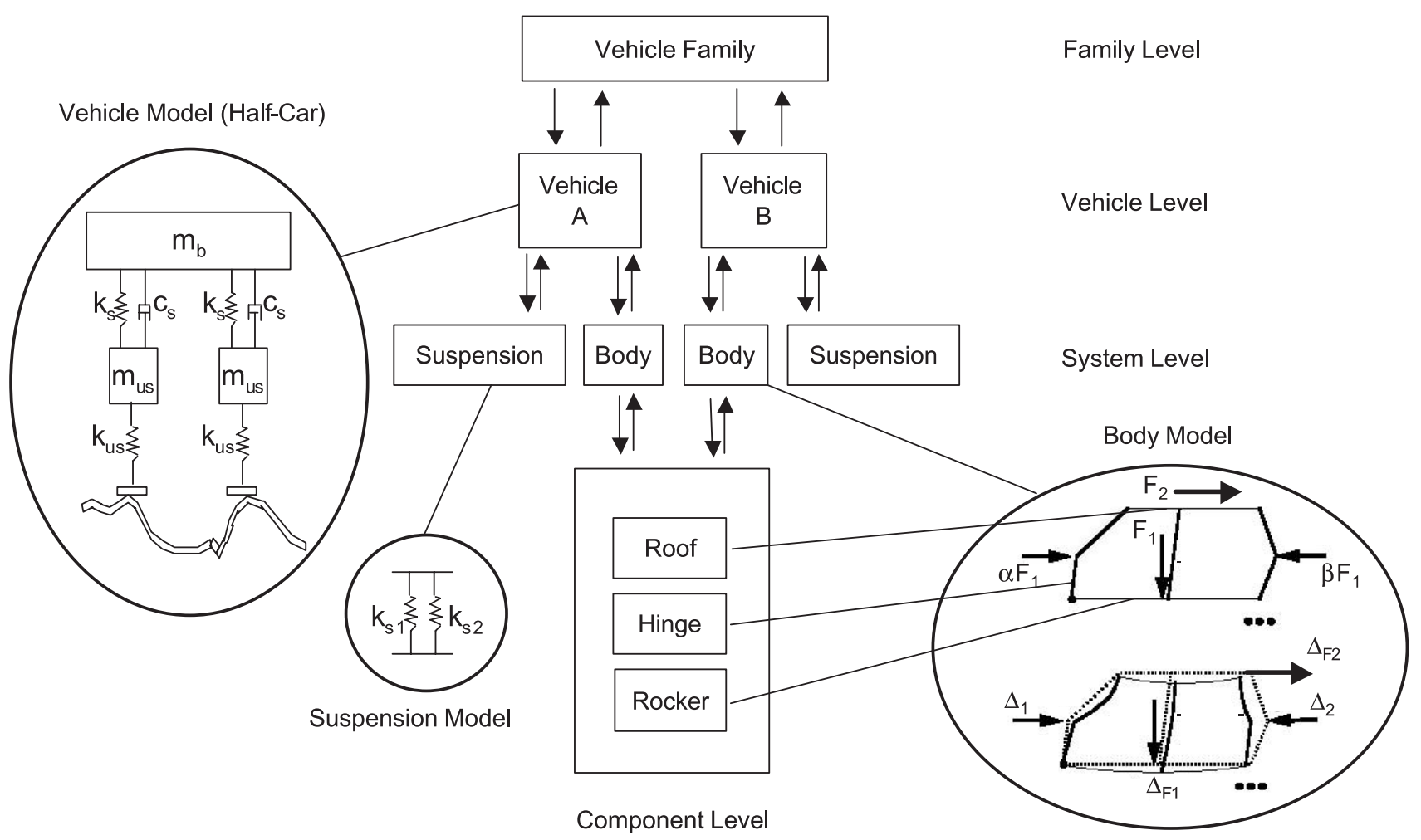

Fig. 3 Model hierarchy

been formulated. The model hierarchy, depicted in Fig. 3, consists of four levels: the family (top) level, the vehicle level, the system level, and the component level. At the family level an objective is defined that combines the masses $m_{v, A}$ and $m_{v, B}$ of two vehicle variants. More emphasis is given to minimizing the mass $m_{v, A}$ of vehicle $\mathrm{A}$.

Figure 4 illustrates the overall target cascading formulation and coordination. The individual vehicles are modelled as half-cars at the vehicle level. In addition to the mass targets $m_{v, A}^{U}$ and $m_{v, B}^{U}$ cascaded from the family level, local targets $\mathbf{T}_{A}$ and $\mathbf{T}_{B}$ are set for the ride quality $\mathbf{z}_{v, A}$ and $\mathbf{z}_{v, A}$ of each variant, respectively. Ride quality is defined by the following five responses: front and rear ride frequency, front and rear wheel hop frequency, and under-steer gradient. Vehicle A should have a stiffer ride, whereas vehicle B should have a softer ride. The halfcar model computes vehicle mass $m_{v}$, ride quality metrics $\mathbf{z}_{v}$, body-in-white mass $m_{b}$, and suspension stiffnesses $k_{s f}$ and $k_{s r}$. Vehicle responses must meet targets determined at the family and system levels (denoted by superscripts ${ }^{U}$ and ${ }^{L}$, respectively) and local targets $\mathbf{T}_{A}$ and $\mathbf{T}_{B}$. Once the responses are computed, they are used as targets at the family and system levels (denoted by superscripts ${ }^{L}$ and ${ }^{U}$, respectively). Note that the subscripts,$A$ and ${ }_{B}$ included above to denote vehicle variants will be omitted in the remainder of the text for simplicity. Local design variables at the system level include distance of centre of gravity (CG) to front end $c g_{f}$, distance of centre of gravity to rear end $c g_{r}$, front tire stiffness $k_{u s f}$, rear tire stiffness $k_{u s r}$, front cornering stiffness $k_{c f}$, and rear cornering stiffness $k_{c r}$.
Body and suspensions are modelled at the system level. The local target $\mathbf{T}_{b}$ for the body is to maximize the stiffness of the structure by minimizing the deflection vector $\delta_{b}$, which is obtained considering the two different loading conditions shown in Fig. 3, subject to allowable strain energy constraints. The body is represented by a finite element model consisting of ten elements. These ten elements model eight components of a two-dimensional body including the $\mathrm{A}, \mathrm{B}$, and $\mathrm{C}$ pillars, the hinge pillar, the roof rail and the rocker. Each component $i$ is described by its cross-sectional properties: footprint area $A_{i}$, real area $A_{R i}$, and moment of inertia $I_{i}$, which are functions of sheet metal thickness $t_{i}$ and height $h_{i}$ and width $b_{i}$ of the footprint area. The joints linking elements are modelled as radial springs. The body model computes body-in-white mass $m_{b}$, deflection $\delta_{b}$, and footprint area $A_{i}$, real area $A_{R i}$, and moment of inertia $I_{i}$ for each body component $i$. The suspension model computes sprung stiffnesses $k_{s f}$ and $k_{s r}$ for the front and rear suspensions of the half-car model, respectively, based on the stiffness of two individual springs $k_{s f 1}$ and $k_{s f 2}$ for the front suspension and two individual springs $k_{s r 1}$ and $k_{s r 2}$ for the rear suspension. System responses must meet targets determined at the vehicle and component levels (denoted by superscripts ${ }^{U}$ and ${ }^{L}$, respectively) and local targets $\mathbf{T}_{b}$. Once the responses are computed, they are used as targets at the vehicle and component levels (denoted by superscripts ${ }^{L}$ and ${ }^{U}$, respectively).

At the component level, each component $i$ of the body comprising the platform is designed to match the area targets $A_{i}^{U}$ and $A_{R i}^{U}$ and moment of inertia target 


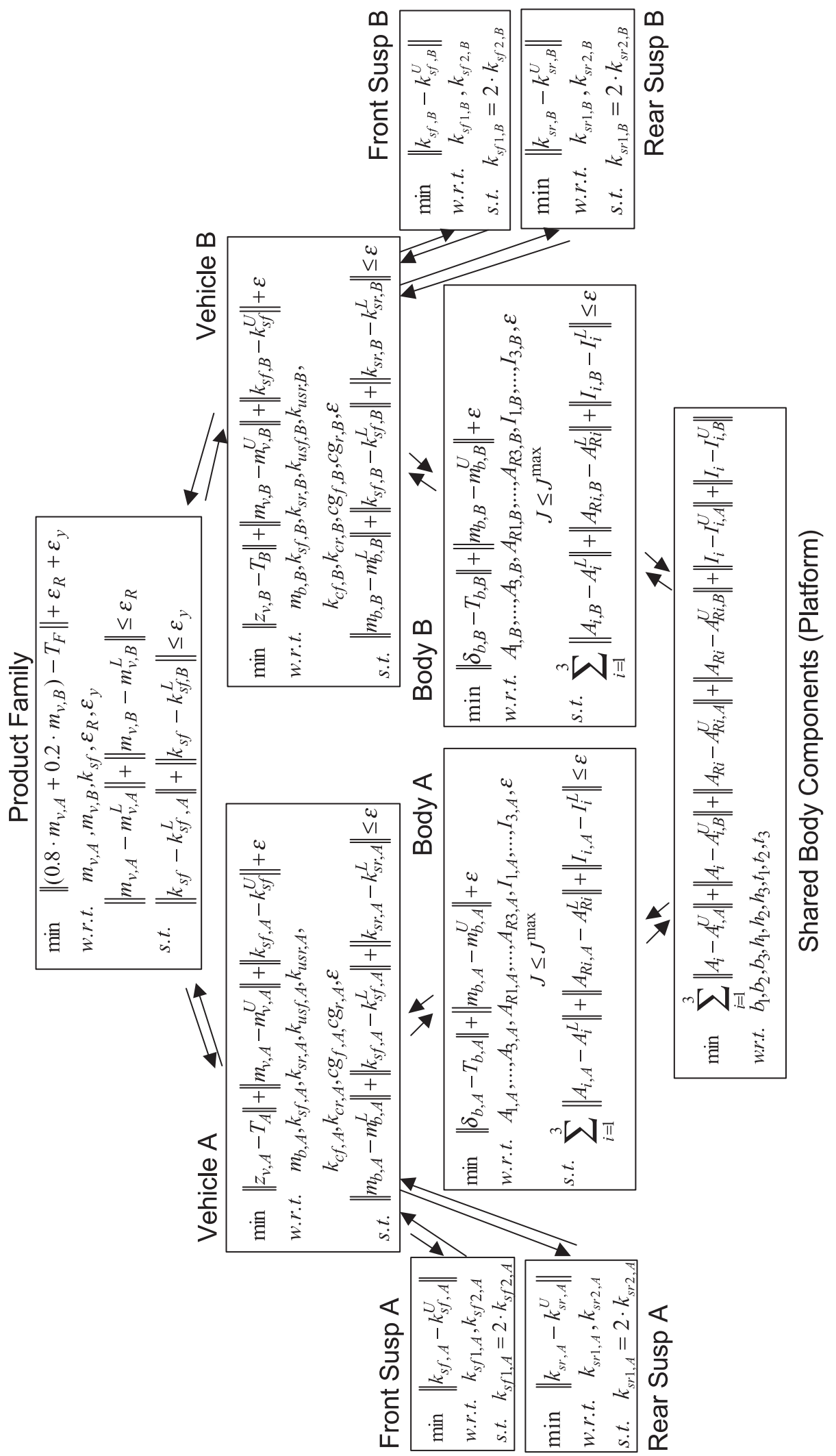

Fig. 4 Target cascading formulation and coordination

$I_{i}^{U}$ cascaded from the system level by determining optimal combinations of cross-sectional dimensions (width $b_{i}$, height $h_{i}$, and thickness $t_{i}$ ). Once these dimensions are found, analytical expressions are evaluated and optimal values $A_{i}^{L}, A_{R i}^{L}$, and $I_{i}^{L}$ are passed to the system level for each platform component $i$. The product platform for the family consists of three body components: namely, the roof, rocker, and hinge pillar. To implement the prod- 
uct platform in the target cascading methodology, one common design problem for the three shared components is formulated at the component level. The shared pillars return a common response to the body models of both vehicle variants.

The front suspension is shared between the two vehicles to illustrate the concept of linking variables and increase the complexity of the case study. This sharing is represented by treating the front suspension stiffness $k_{s f}$ at the vehicle level as a linking variable. This linking variable is coordinated at the family level by computing the suspension stiffness $k_{s f}$ to match the values $k_{s f, A}^{L}$ and $k_{s f, B}^{L}$ determined at the vehicle level for each variant. The computed value is then cascaded to both variants at the vehicle level as $k_{s f}^{U}$. Note that the front suspension stiffness is also treated as a response at the vehicle level that is cascaded as target to the system level.

To solve the multilevel problem a generic coordination strategy is implemented that starts at the top-most level. Each level is solved in sequence, and then the problems are solved once again by returning to the top level problem. This process is counted as one iteration, and convergence is tested by checking if deviation terms are sufficiently reduced.
5

\section{Results and discussion}

As mentioned above, the family objective was to minimize the weighted sum of the masses of the two vehicles. The target was set to zero in order to achieve the minimum mass possible. Of course, such a target is unattainable, and the optimal value found was $2124 \mathrm{~kg}$. The target cascading process converged after ten iterations. The optimal values obtained for the responses for which targets were defined locally at the vehicle level are presented in Table 1.

It can be seen that all targets are met with a satisfactory accuracy, except for the front ride frequency. Indeed, sharing the front suspension results into the inability to satisfy this target. Therefore, it is necessary to either define another set of target values or reconsider the sharing of the front suspension.

Local targets were also set for the bodies at the system level. Bodies were intended to be as stiff as possible by minimizing the deflection vector $\delta_{b}$. Once again, unattainable zero targets were set and the following values were obtained: The component of the deflection vector due to vertical loading is 0.209 and 0.202 inches for

Table 1 Target and optimal values for vehicle level responses

\begin{tabular}{llccc}
\hline Responses $\mathbf{z}_{v}$ & $\begin{array}{l}\text { Target value } \\
\text { Vehicle A }\end{array}$ & $\begin{array}{l}\text { Optimal value } \\
\text { Vehicle A }\end{array}$ & $\begin{array}{l}\text { Target value } \\
\text { Vehicle B }\end{array}$ & $\begin{array}{l}\text { Optimal value } \\
\text { Vehicle B }\end{array}$ \\
\hline \hline Front ride frequency $[\mathrm{Hz}]$ & 1.273 & 1.160 & 0.955 & 1.120 \\
Rear ride frequency $[\mathrm{Hz}]$ & 1.592 & 1.585 & 1.592 & 1.592 \\
Front wheel hop frequency $[\mathrm{Hz}]$ & 10.345 & 10.348 & 10.345 & 10.343 \\
Rear wheel hop frequency $[\mathrm{Hz}]$ & 10.345 & 10.347 & 9.549 & 9.549 \\
Under-steer gradient $\left[\mathrm{rad} / \mathrm{m} / \mathrm{s}^{2}\right]$ & $7.19 \times 10^{-3}$ & $7.186 \times 10^{-3}$ & $7.19 \times 10^{-3}$ & $7.191 \times 10^{-3}$ \\
\hline
\end{tabular}

Table 2 Vehicle responses and linking variable values computed at the family and vehicle levels

\begin{tabular}{llrr}
\hline Characteristic & Type & Family level value & Vehicle level value \\
\hline \hline Mass of vehicle A $m_{v, A}[\mathrm{~kg}]$ & Response & 2139 & 2139 \\
Mass of vehicle B $m_{v, B}[\mathrm{~kg}]$ & Response & 2162 & 2163 \\
Front suspension stiffness of vehicle A $k_{s f, A}[\mathrm{~N} / \mathrm{mm}]$ & Linking variable & 35.400 & 35.490 \\
Front suspension stiffness of vehicle B $k_{s f, B}[\mathrm{~N} / \mathrm{mm}]$ & Linking variable & 35.400 & 35.500 \\
\hline
\end{tabular}

Table 3 System responses computed at the vehicle and system levels

\begin{tabular}{lcc}
\hline Response & Vehicle level value & System level value \\
\hline \hline Front suspension stiffness of vehicle A $k_{s f, A}[\mathrm{~N} / \mathrm{mm}]$ & 35.490 & 35.499 \\
Front suspension stiffness of vehicle B $k_{s f, B}[\mathrm{~N} / \mathrm{mm}]$ & 35.500 & 35.499 \\
Rear suspension stiffness of vehicle A $k_{r f, A}[\mathrm{~N} / \mathrm{mm}]$ & 39.860 & 39.790 \\
Rear suspension stiffness of vehicle B $k_{r f, B}[\mathrm{~N} / \mathrm{mm}]$ & 36.560 & 36.617 \\
Body-in-white mass of vehicle A $m_{b}, A[\mathrm{~kg}]$ & 240 & 239 \\
Body-in-white mass of vehicle B $k_{b}, B[\mathrm{~kg}]$ & 263 & 263 \\
\hline
\end{tabular}


Table 4 Platform component responses computed at the system and component levels

\begin{tabular}{lcrr}
\hline Response & $\begin{array}{l}\text { System level value } \\
\text { vehicle A }\end{array}$ & $\begin{array}{l}\text { System level value } \\
\text { vehicle B }\end{array}$ & Component level value \\
\hline \hline Moment of inertia of rocker $I_{1}\left[\mathrm{in}^{4}\right]$ & 15.387 & 15.384 & 15.387 \\
Footprint cross-sectional area of rocker $A_{1}\left[\mathrm{in}^{2}\right]$ & 8.024 & 8.031 & 8.020 \\
Real cross-sectional area of rocker $A_{R 1}\left[\mathrm{in}^{2}\right]$ & 5.788 & 5.788 & 5.792 \\
Moment of inertia of roof rail $I_{2}\left[\mathrm{in}^{4}\right]$ & 0.162 & 0.161 & 0.157 \\
Footprint cross-sectional area of roof rail $A_{2}\left[\mathrm{in}^{2}\right]$ & 1.548 & 1.542 & 1.550 \\
Real cross-sectional area of roof rail $A_{R 2}\left[\mathrm{in}^{2}\right]$ & 1.043 & 1.044 & 1.043 \\
Moment of inertia of hinge pillar $I_{3}\left[\mathrm{in}^{4}\right]$ & 13.917 & 8.177 & 13.887 \\
Footprint cross-sectional area of hinge pillar $A_{3}\left[\mathrm{in}^{2}\right]$ & 8.152 & 5.888 & 8.158 \\
Real cross-sectional area of hinge pillar $A_{R 3}\left[\mathrm{in}^{2}\right]$ & 5.879 & 5.883 \\
\hline
\end{tabular}

Table 5 Optimal values of local design variables at the vehicle, system, and component levels

\begin{tabular}{|c|c|c|c|}
\hline Design variable & Level & $\begin{array}{l}\text { Optimal value } \\
\text { vehicle } \mathrm{A}\end{array}$ & $\begin{array}{l}\text { Optimal value } \\
\text { vehicle B }\end{array}$ \\
\hline Distance of CG to front end $c g_{f}[\mathrm{~m}]$ & Vehicle & 1.390 & 1.250 \\
\hline Distance of CG to rear end $c g_{r}[\mathrm{~m}]$ & Vehicle & 2.310 & 2.450 \\
\hline Front tire stiffness $k_{u s f}[\mathrm{~N} / \mathrm{mm}]$ & Vehicle & 24.10 & 24.08 \\
\hline Rear tire stiffness $k_{u s r}[\mathrm{~N} / \mathrm{mm}]$ & Vehicle & 24.09 & 20.52 \\
\hline Front cornering stiffness $k_{c f}\left[\mathrm{~N} / \mathrm{rad} / 10^{-4}\right]$ & Vehicle & 10.47 & 11.30 \\
\hline Rear cornering stiffness $k_{c r}\left[\mathrm{~N} / \mathrm{rad} / 10^{-4}\right]$ & Vehicle & 12.82 & 11.84 \\
\hline Front suspension spring stiffness $k_{s f 1}[\mathrm{~N} / \mathrm{mm}]$ & System & 23.666 & 23.673 \\
\hline Front suspension spring stiffness $k_{s f 2}[\mathrm{~N} / \mathrm{mm}]$ & System & 11.833 & 11.836 \\
\hline Rear suspension spring stiffness $k_{s r 1}[\mathrm{~N} / \mathrm{mm}]$ & System & 26.526 & 24.411 \\
\hline Rear suspension spring stiffness $k_{s r 2}[\mathrm{~N} / \mathrm{mm}]$ & System & 13.263 & 12.206 \\
\hline Width of rocker cross-section $b_{1}[\mathrm{in}]$ & Component & 1.510 & shared \\
\hline Height of rocker cross-section $h_{1}$ [in] & Component & 5.310 & shared \\
\hline Thickness of rocker cross-section $t_{1}[\mathrm{in}]$ & Component & 0.497 & shared \\
\hline Width of roof rail cross-section $b_{2}$ [in] & Component & 1.335 & shared \\
\hline Height of roof rail cross-section $h_{2}$ [in] & Component & 1.161 & shared \\
\hline Thickness of roof rail cross-section $t_{2}$ [in] & Component & 0.265 & shared \\
\hline Width of hinge pillar cross-section $b_{3}$ [in] & Component & 1.642 & shared \\
\hline Height of hinge pillar cross-section $h_{3}$ [in] & Component & 4.969 & shared \\
\hline Thickness of hinge pillar cross-section $t_{3}$ [in] & Component & 0.530 & shared \\
\hline
\end{tabular}

vehicles $\mathrm{A}$ and $\mathrm{B}$, respectively; the component of the deflection vector due to horizontal loading is 0.357 and 0.358 inches for vehicles $\mathrm{A}$ and $\mathrm{B}$, respectively.

Responses and linking variable values at the family and vehicle levels are compared in Table 2 . The agreement is satisfactory. Note that the front suspension stiffness is treated as a linking variable during the coordination process between these two levels.

The matching of responses between vehicle and system levels is illustrated in Table 3. Once again, deviations are negligible. Note that during the coordination of these two levels the front suspension stiffness is treated as a response. Tables 2 and 3 show that the target cascading process forces a consistent design.

Table 4 presents the results obtained for the product platform, i.e. the three components of the body that are common to the two vehicles. The agreement between the values obtained at the system level and the values ob- tained at the component level confirms the ability of the target cascading formulation to account for shared components.

Finally, Table 5 presents the optimal values for local design variables for the vehicle, system, and component levels.

Although design values are obtained for all optimization problems formulated within the target cascading formulation, it should be emphasized that the main outcome of this process are the design specifications for the elements of the variants at the vehicle, system, and component levels; namely, vehicle masses, body-in-white masses, suspension stiffnesses, and cross-section related properties (areas and moments of inertia) for the platform components of the body. These design specifications correspond to the optimal values of the responses, as presented in the far-right columns of Tables 2-4. For example, the design specification for the mass of vehicle B is $2163 \mathrm{~kg}$, the de- 
sign specification for the body-in-white mass of vehicle A is $239 \mathrm{~kg}$, and the design specification for the footprint area of the rocker is $8.02 \mathrm{in}^{2}$.

\section{6 \\ Conclusions}

The target cascading methodology for optimal product development has been extended to the design of product families. The single-product formulation has been modified to accommodate the presence of shared elements and locally introduced targets. Given a platform, hierarchical partitions of the individual product design problems, and the necessary analysis models, family and product targets are cascaded down to systems, subsystems, and components. In this manner design specifications are determined for all elements, including the product platform, and a consistent design is obtained. Moreover, tradeoffs between commonality and target achievement can be identified. The information flow within the coordination strategy is based on the hierarchical multilevel structure underlying the family design problem. The technique was successfully applied to an automotive example of two vehicles that share the front suspension and a number of body components. Application to more complicated examples is straightforward albeit demanding with respect to appropriate element models.

Acknowledgements This research was partially supported by the Automotive Research Center (ARC), a US Army Center of Excellence in Modeling and Simulation of Ground Vehicles at the University of Michigan, and by a URP grant from Ford Motor Company. The authors would also like to thank Noboru Kikuchi for providing the finite element body model.

\section{References}

Conner, C.G.; De Kroon, J.P.; Mistree, F. 1999: A product variety evaluation method for a family of cordless drill transmissions. Proc. ASME Design Engineering Technical Conf. (held in Las Vegas, NV), Paper No. DETC99/DAC-8625

Ericsson, A.; Erixon, G. 1999: Controlling design variants: modular product platforms. ASME Press

Fellini, R.; Papalambros, P.Y.; Weber, T. 2000: Application of a product platform design process to automotive powertrains. Proc. 8-th AIAA/USAF/NASA/ISSMO Symp. on Multidisciplinary Analysis and Optimization (held in Long Beach, CA), Paper No. 4849

Fujita, K.; Akagi, S.; Yoneda, T.; Ishikawa, M. 1998: Simultaneous optimization of product family sharing system structure and configuration. Proc. ASME Design Engineering Technical Conf. (held in Atlanta, GA), Paper No. DETC98/ DFM-5722
Gonzalez-Zugasti, J.P.; Otto, K.N.; Baker, J.D. 1998: A method for architecting product platforms with an application to interplanetary mission design. Proc. ASME Design Engineering Technical Conf. (held in Atlanta, GA), Paper No. DETC98/DAC-5608

Gonzalez-Zugasti, J.P.; Otto, K.N. 2000: Modular platformbased product family design. Proc. ASME Design Engineering Technical Conf. (held in Baltimore, MA), Paper No. DETC2000/DAC-14238

Kim, H.M. 2001: Target cascading in optimal system design. Ph.D. Thesis, University of Michigan

Kim, H.M.; Michelena, N.; Papalambros, P.Y.; Jiang, T. 2000: Target cascading in optimal system design. 26-th Design Automation Conf. (held in Baltimore, MD), Paper No. 14265

Kota, S.; Sethuraman, K. 1998: Managing variety in product families through design for commonality. Proc. ASME Design Engineering Technical Conf. (held in Atlanta, GA), Paper No. DETC98/DTM-5651

Martin, M.; Ishii, K. 1997: Design for variety: development of complexity indices and design charts. Proc. ASME Design Engineering Technical Conf. (held in Sacramento, CA), Paper No. DETC97/DFM-4359

Martin, M.; Ishii, K. 2000: Design for variety: a methodology for developing platform architectures. Proc. ASME Design Engineering Technical Conf. (held in Baltimore, MD), Paper No. DETC2000/DFM-14021

McGrath, M.E. 1995: Product strategy for high-technology companies. New York: Irwin Professional Publishing

Meyer, M.H.; Lehnerd, A.P. 1997: The power of product platforms. New York: Free Press

Michelena, N.F.; Papalambros, P.Y.; Park, H.A.; Kulkarni, D. 1999: Hierarchical overlapping coordination for large-scale optimization by decomposition. AIAA J. 37, 890-896

Michelina, N.F.; Park, H.A.; Papalambros, P.Y. 2002: Convergence properties of analytical target cascading. Proc. 9th AIAA/ISSMO Symp. on Multidiscipinary Analysis and Optimization (held in Atlanta, GA)

Nelson, S.A.; Parkinson, M.B.; Papalambros, P.Y. 1999: Multicriteria optimization in product platform design. Proc. 25-th Design Automation Conf. (held in Las Vegas, NV), Paper No. 8676

Siddique, Z.; Rosen, D.W.; Wang, N. 1998: On the applicability of product variety design concepts to automotive platform commonality. Proc. ASME Design Engineering Technical Conf. (held in Atlanta, GA), Paper No. 98-DETC/DTM5661

Simpson, T.W.; Maier, J.R.A.; Mistree, F. 1999: A product platform concept exploration method for product platform design. Proc. ASME Design Theory and Methodology Conf. (held in Las Vegas, NV), Paper No. DETC99/DTM-8761

Ulrich, K.T.; Eppinger, S.D. 1995: Product design and development. New York: McGraw-Hill 